\title{
Ishak Score 6
}

National Cancer Institute

\section{Source}

National Cancer Institute. Ishak Score 6. NCI Thesaurus. Code C95156.

Marked fibrosis with cirrhosis is present. 